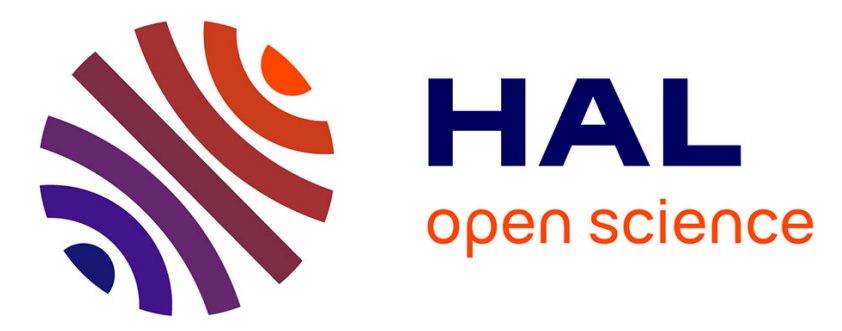

\title{
Interactive RGB transparency: A color rendering tool for superimposed translucent layers in digital images
}

\author{
Lionel Simonot, Mathieu Hébert
}

\section{To cite this version:}

Lionel Simonot, Mathieu Hébert. Interactive RGB transparency: A color rendering tool for superimposed translucent layers in digital images. IS\&T International Symposium on Electronic Imaging 2018, Material Appearance 2018, Jan 2018, Burlingame, United States. 10.2352/ISSN.24701173.2018.8.MAAP-167 . hal-01700606

\section{HAL Id: hal-01700606 https://hal.science/hal-01700606}

Submitted on 5 Feb 2018

HAL is a multi-disciplinary open access archive for the deposit and dissemination of scientific research documents, whether they are published or not. The documents may come from teaching and research institutions in France or abroad, or from public or private research centers.
L'archive ouverte pluridisciplinaire HAL, est destinée au dépôt et à la diffusion de documents scientifiques de niveau recherche, publiés ou non, émanant des établissements d'enseignement et de recherche français ou étrangers, des laboratoires publics ou privés. 


\title{
Interactive RGB Transparency: a color rendering tool for superimposed translucent layers in digital images
}

\author{
Lionel Simonot; Université de Poitiers, Institut Prime UPR CNRS 3346; Futuroscope Chasseneuil, France \\ Mathieu Hébert; Univ Lyon, UJM-Saint-Etienne, CNRS, Institut d Optique Graduate School, Laboratoire Hubert Curien UMR 5516, F- \\ 42023, SAINT-ETIENNE, France
}

\begin{abstract}
Interactive RGB Transparency is an open source tool dedicated to the visualization of transparency effects in digital color images. Transparency effect can be rendered as a binary color mixing between foreground and background layers, which can be uniformly colored layers or downloaded images, while the transparency rate can be modified interactively from perfectly transparent to perfectly opaque. The tool also allows the inverse operation, by removing transparency effects in an image. While most common softwares render transparency effects by additive color mixing (eventually subtractive color mixing), Interactive $R G B$ Transparency proposes three different approaches: i) defining new color mixing laws varying continuously from additive to subtractive color mixing, ii) defining color mixing as a generalized average between the colors of the layers, iii) considering a translucent scattering layer whose thickness can be modified.
\end{abstract}

\section{Introduction}

In contrast with color, transparency is a visual appearance attribute of second order, perceived by analyzing the color transformation of a scene in background by an object in front of it. Transparency is physically based on the capacity of the object to transmit light from the background without scattering it, therefore to transmit a rather sharp image of the scene even though its colors are modified. However, physical transparency is neither a sufficient nor a necessary condition for perceptual transparency. It is possible to create transparence illusions in still images, by coloring some areas in such manner that they will be perceived as forming a transparent object in front of a background. The artist Josef Albers, known as a student and professor at Bauhaus, studied the perception of juxtaposed colors and showed, in his book Interaction of color [1], how to create in practice the illusion of transparency by using only a set of opaque painted papers. In a more formal way, Metelli [2] states that the transparency illusion is generated under several conditions. Among them, the colorimetric changes between the surfaces represented with and without transparency must be coherently interpreted as transparency by the brain [3]. The color transparency can be studied as a binary color mixing between foreground and background layers. The colorimetric coherence is possible if the same mixing law is applied for all points of the foreground layer assumed to be transparent.

Many softwares including color features (text, image processing, presentation, drawing, etc.) enable transparency effects. But most of them render transparency by additive color mixing law, i.e., by linear combination of the RGB values of the different layers or images, which is very restrictive. It actually renders what physically occurs when a partially pierced opaque surface, like a mesh, is seen in front of a scene (Figure 1, on the left). But it cannot generate other transparency effects such as a scene viewed through a colored filter (Figure 1, on the right).

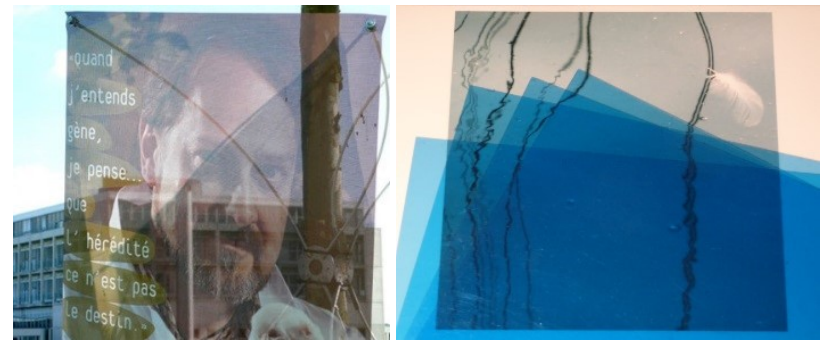

Figure 1. Illustration of additive transparency with an advertisement poster printed on net curtain (on the left), and of subtractive transparency with a stack of blue transparent acetate sheets on a photograph (on the right).

In order to widen the range of translucency effects that can be rendered, we developed an open source tool, Interactive $R G B$ Transparency, dedicated to the display of transparency effects in digital color images by offering different translucency computation methods. The background and foreground can each one be either a uniform color or any downloaded image, while the transparency rate and transparency type can be modified interactively thanks to various adjustable parameters. The inverse operation is also enabled, i.e., a transparency effect can be removed from a given image.

The tool relies on three different concepts regarding transparency, illustrated in Figure 2:

- From additive to subtractive transparency

In color images, transparency effect is rendered by combining the RGB values of the superimposed layers. The combination relies on color mixing laws presented in Ref. [4], forming a continuous set of laws between the purely additive law ("additive transparency") and the purely subtractive law ("subtractive transparency"), tuned thanks to an adjustable parameter.

- Transparency as a generalized f-mean

Transparency can be described as a weighted mean between the RGB values of the superimposed layers. While the purely additive and purely subtractive transparencies correspond to the arithmetic and geometric mean respectively, we propose a generalization by the use of a weighted generalized $f$-mean including the arithmetic and geometric means but also the harmonic and quadratic means, as well as the Kubelka-Munk function.

- Translucency by a scattering layer

The prediction of the color appearance due to a translucent layer placed in front of a background requires taking into account the multiple reflections between the scattering layer and the background. The description of the scattering layer is defined by the reflectance images for the corresponding layers with infinite thickness (opaque foreground layer) and with unit thickness. The unit thickness layer reflectance is described as the combination of the opaque foreground layer and an achromatic component. The transparency rate is adjusted by the relative thickness of the layer. 


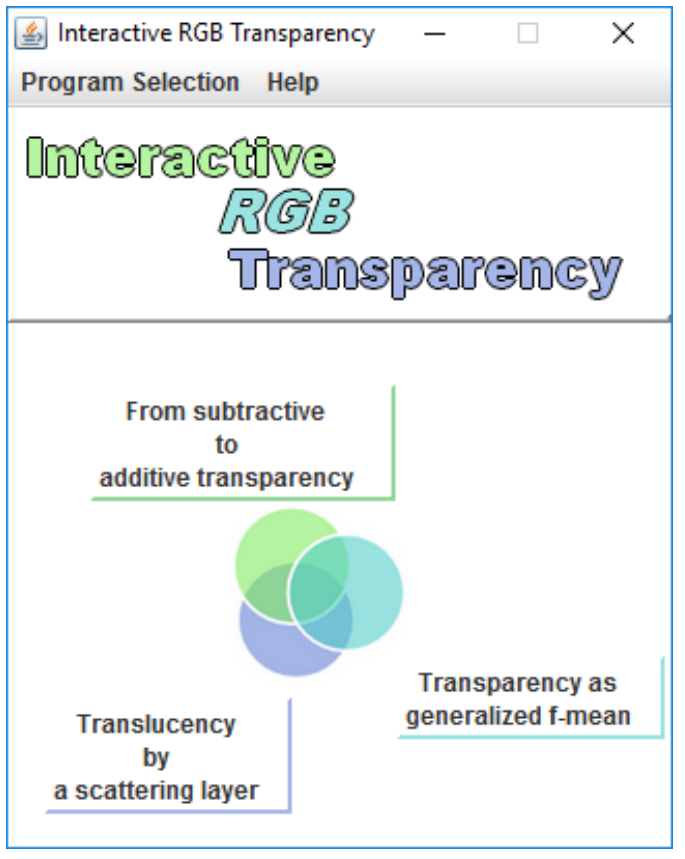

Figure 2. First window after launching the software: the user must choose between three approaches.

The software interface is shortly described in Section 2. Some generalities about color mixing are presented in Section 3 and a detailed description of the three approaches in Section 4. In Section 5 , the inverse transparency principles are given.

\section{Software interface}

Interactive $R G B$ Trasmparency is an open source software that can be freely downloaded [5].

Figure 2 shows the first window after launching the software. The user can choose one of the three approaches to render transparency. For each approach, similar visualization window is displayed, as presented in Figure 3.

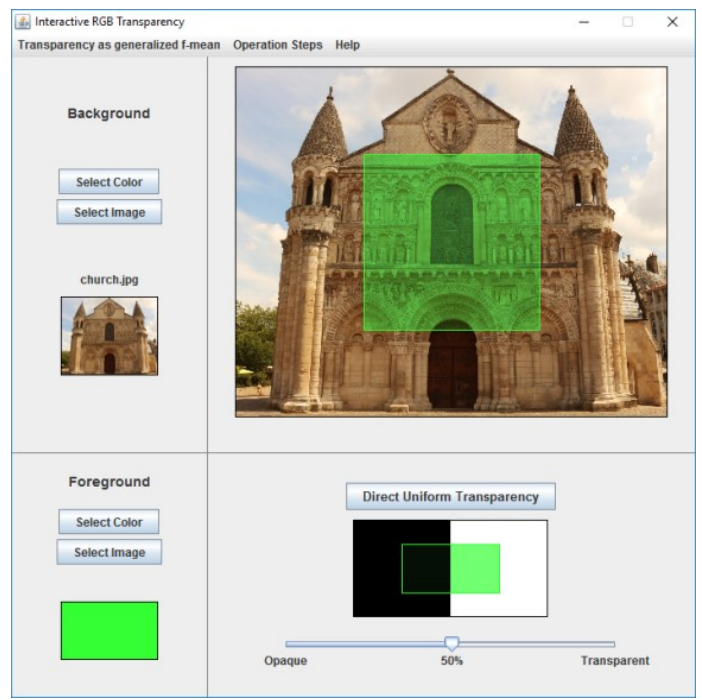

Figure 3. Visualization window: for a given transparency law, the user can choose the background, the foreground and the transparency rate.
For a given transparency law, the user can choose the background and the foreground layers, each one being either a uniform color layer or a downloaded image. The transparency rate can be also modified interactively from $0 \%$ for an opaque foreground to $100 \%$ where the foreground disappears. The result is displayed for the selected foreground image or color on a contrast card, juxtaposition of a perfectly black and a perfectly white background. The contrast cards are traditionally used in the ink and painting formulation industry, in particular to control the minimum layer thickness needed to obtain the opacity. All possible settings offered by the software are described in details in the user manual

\section{Generalities on color mixing laws}

Transparency can be considered as a binary color mixing between the foreground layer (labeling subscript $f$ ) and the background layer (subscript $g$ ). A color is defined here as a reflectance spectrum $x(\lambda)$ satisfying

$0<x(\lambda)<1 \quad \forall \lambda \in$ visible range

The number of wavebands depends on the measurement system. At least 3 wavebands in the visible range are needed to define colors but it may be larger, or much larger in the cases of multi- and hyperspectral systems. All color mixing laws presented in this article can be applied with any number of spectral bands, but we focus here on classical computing systems where 24-bit colors are stored as 3 integers $X=R, G, B$ between 0 and 255 . They are then considered as the reflectance for three distinct wavelengths or spectral bands. To ensure the property given by Eq. (1), the reduced coordinates $x=r, g, b$ are calculated as follows:

$x=\frac{253 X+255}{255^{2}}$

The color transparency exists if the same function $h$ is applied to every point of the transparent foreground layer, and every wavelength:

$$
x=h\left(x_{f}, x_{g}, c\right)
$$

where $x_{f}, x_{g}$ and $x$ are the foreground, background and resulting reflectances respectively. The transparency rate $c$ can vary from 0 for an opaque foreground to 1 in absence of foreground. Therefore, the resulting reflectance $x$ varies from $x_{f}$ for $c=0$ to $x_{g}$ for $c=1$. We restrict the study to functions $h$ varying as a monotonous function of the transparency rate $c$, thus avoiding that one reflectance value can be obtained from different transparency rates, which would induce confusion in the transparency perception.

Figure 4 presents the flowchart explaining the binary color mixing computation in the case of RGB values.

As a typical example, the additive transparency corresponds to a scene seen through a partially pierced surface, the holes occupying a fraction $c$ of the foreground image. The additive mixing law is a linear combination between the foreground and background reflectances:

$x=(1-c) x_{f}+c x_{g}$ 
Selection by the users

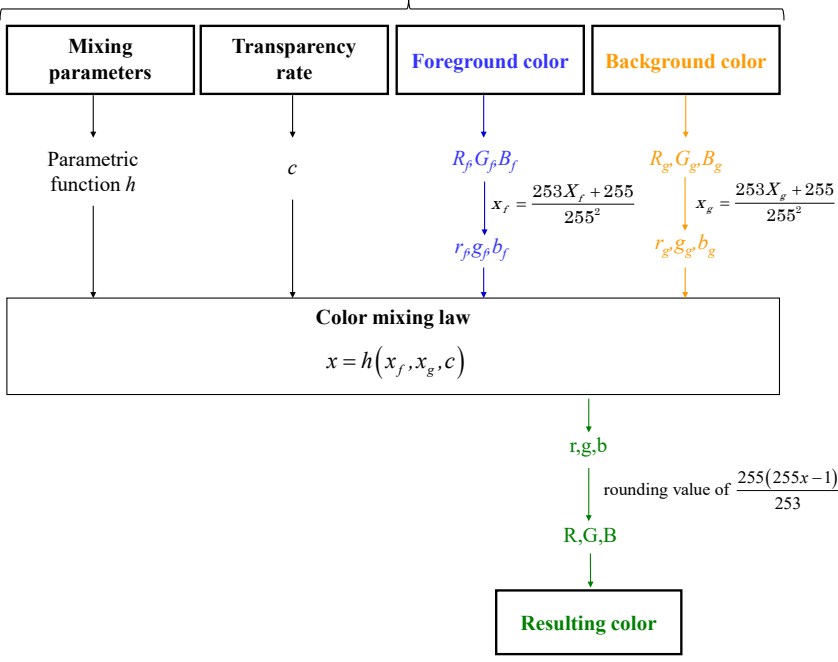

Figure 4. Flowchart for the color mixing calculation from RGB images applied to each pixel of the images.

The subtractive transparency corresponds to a scene seen through a colored glass. The purely subtractive mixing law consists in a weighted geometric mean of the foreground and background reflectances:

$x=x_{f}^{1-c} x_{g}^{c}$

It is worth noting that if $x_{f}$ or $x_{g}$ is zero, the resulting reflectance $x$ would be equal to zero for any transparency rate. This discontinuity may yield an aberrant color for the subtractive mixing. That is the reason why we restrict the reflectance values to be strictly positive. In the condition (1), we also exclude the maximum value $x=1$ for other mixing laws presented hereafter. In practice, these extreme values are replaced by $1 / 255$ and $254 / 255$ by using the transformation (2).

\section{Three approaches}

By applying the generalities presented in the previous section, we develop three approaches (cf. Figure 2) in order to define color mixing law families.

\subsection{From subtractive to additive transparency}

In this first approach introduced in Ref. [4], two simple empirical models are proposed to create intermediate configurations between purely additive and purely subtractive color mixings. An additional parameter $\tau$ is introduced to tune the proportions of additive and subtractive mixings. The additive-subtractive law can be written as

$$
x=\tau\left((1-c) x_{f}+c x_{g}\right)+(1-\tau) x_{f}^{1-c} x_{g}^{c}
$$

while the subtractive-additive law is expressed as

$x=\left((1-c) x_{f}^{\tau}+c x_{g}^{\tau}\right) x_{f}^{(1-c)(1-\tau)} x_{g}^{c(1-\tau)}$
For both mixing laws [Eqs. (6) and (7)], the mixing parameter $\tau$ varies from 0 for the ideal subtractive mixing [Eq. (5)] to 1 for the ideal additive mixing [Eq. (4)]. Figure 5a-c shows examples for $\tau=0,0.5$ and 1 . The subtractive law gives darker and often more saturated colors than the additive law. The contrast of the resulting image is also better with the subtractive law.
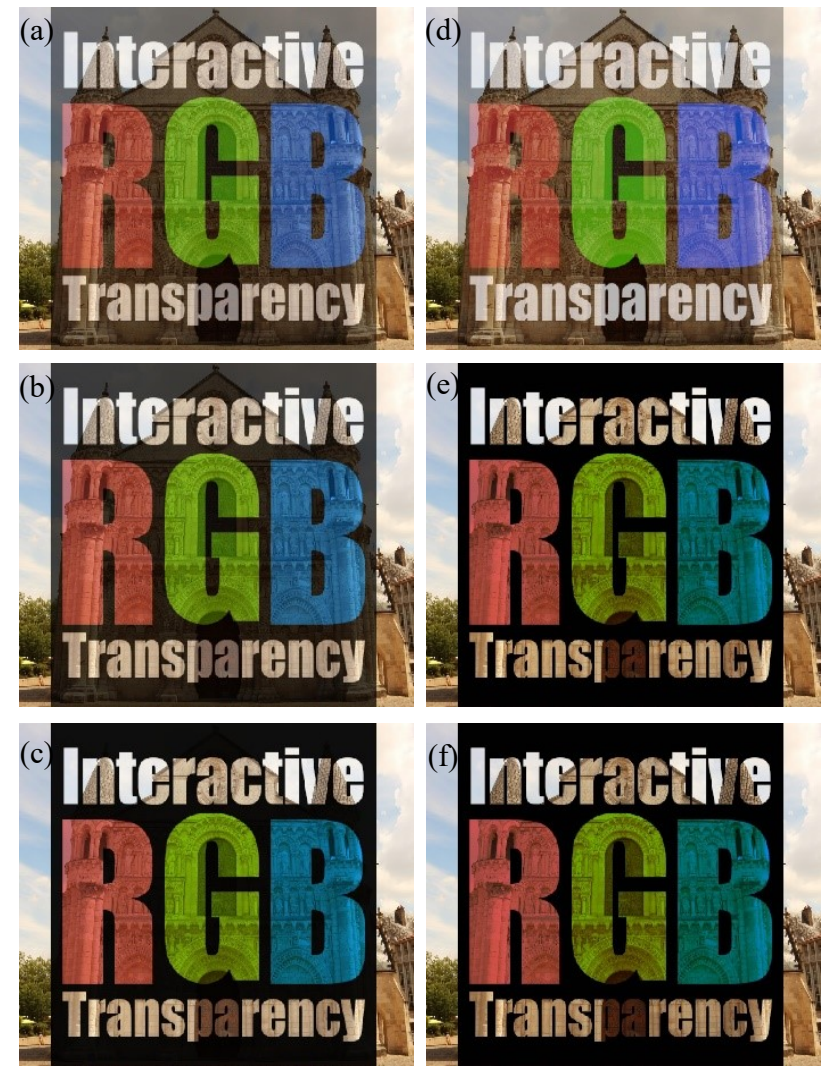

Figure 5. Different transparency effects (transparency rate $c=0.5$ ).

(a) Additive mixing, (b) additive-subtractive mixing with $\tau=0.5$, (c) subtractive mixing, (d) weighted quadratic mean, (e) weighted harmonic mean,

(f) weighted mean with Kubelka-Munk function.

\subsection{Transparency as a generalized f-mean}

The additive mixing law [Eq. (4)] and the subtractive mixing law [Eq. (5)] correspond to the weighted arithmetic and geometric means, respectively. We propose a generalization by the use of a weighted generalized $f$-means, defined as

$x=f^{-1}\left[(1-c) f\left(x_{f}\right)+c f\left(x_{g}\right)\right]$

where $f(x)$ is a continuous and injective function for $x \in] 0,1[$. The inversion of function $f$ can be performed numerically or expressed analytically. We first propose the power function:

$$
f(x)=x^{p}
$$

where $p$ can take any real value.

The weighted power mean includes as special cases the additive color mixing for $p=1$ (Figure 5a) as well as the subtractive color 
mixing for $p \rightarrow 0$ (Figure $5 \mathrm{c}$ ). For this latter case, it can be shown that $f(x)=\ln x$. It also includes the weighted quadratic mean (also called root mean square) for $p=2$ (Figure $5 \mathrm{~d}$ ), or the weighted harmonic mean for $p=-1$ (Figure 5e). Surprisingly, the empirical weighted harmonic mean seems to be a better solution than the subtractive mixing law in order to create transparency illusion of a scene viewed through a colored glass. This may be due to the very rough spectral sampling. With only three spectral bands, the geometric mean is not sufficient to precisely simulate the increase of color saturation.

When $p \rightarrow-\infty$ or $p \rightarrow+\infty$, equation (8) tends towards $x=\min \left(x_{f}, x_{g}\right)$ or $x=\max \left(x_{f}, x_{g}\right)$, respectively, for any transparency rate value $c \in] 0,1[$.

For $p=1 / n$, equation (8) can be written as

$$
x=\left[(1-c) x_{f}^{1 / n}+c x_{g}^{1 / n}\right]^{n}
$$

Equation (10) corresponds to the Yule-Nielsen model [6], or its spectral version [7], in the particular case of two primaries (corresponding to the foreground and the background reflectances). This model is classically used to predict halftone colors produced by printing. From the additive mixing law (corresponding to $n=1$ ), the Yule-Nielsen model introduces the corrective parameter $n$ to take empirically into account the lateral propagation of light within the paper bulk and the internal reflections at the paper-air interface. Whereas the typical values of $n$ are found between 1 and some units, Lewandowski et al. [8] mention the possibility to extend the model for negative values of $n$.

We also propose the following function family:

$$
f(x)=\frac{x^{p}}{(1-x)^{q}}
$$

with $p$ and $q$ can take any real value.

The interest of this function is first that it includes as a special case the power function when $q=0$. Moreover, the extreme reflectance values $x=0$ and $x=1$ have a symmetrical importance in expression (11). As the function is not analytically invertible in the general case, we focus on some particular values for $p$ and $q$. The case $p=-1$ and $q=-2$ corresponds to the Kubelka-Munk function [9]. For an opaque scattering layer characterized by its absorption coefficient $K$ and backscattering coefficient $S$, the Kubelka-Munk function relies the ratio $K / S$ to the reflectance $x$ of this layer:

$$
\frac{K}{S}=f_{K M}(x)=2 \frac{(1-x)^{2}}{x}
$$

By introducing the function $f_{K M}$ in the general equation (8), we obtain:

$$
x=f_{K M}^{-1}\left((1-c) f_{K M}\left(x_{f}\right)+c f_{K M}\left(x_{g}\right)\right)
$$

The factor 2 in expression (12) has no influence in the relation (13) due to the compensation of a factor $1 / 2$ in the inverse function. Expression (13) corresponds to a binary color mixing law called as the "one constant method". This method is applied for color prediction in painting industries when the paint scattering is dominated by a single pigment, typically the white one [10]. Figure 5 fillustrates the application of this method for transparency illusion. The resulting image is not identical but close to those obtained with the weighted harmonic mean (Figure 5e).

\subsection{Translucency by a scattering layer}

All previous color mixing laws verify the following property:

$x=h\left(x_{f}, x_{g}, c\right)=h\left(x_{g}, x_{f}, 1-c\right)$

Therefore, it is not possible to distinguish whose layer is the foreground or the background. Relation (14) is not valid with the third approach that we propose now by considering a scattering translucent layer on an opaque background. In this case, the layer thickness $N$ is negatively proportional to the logarithm of the transparency rate $c$. An opaque foreground $(c=0)$ is obtained for an infinite thickness $(N \rightarrow+\infty)$ while the absence of foreground $(c=1)$ corresponds to $N=0$.

For a sake of clarity, we change the notations. We use $r$ (instead of $x$ ), $r_{g}$ (instead of $x_{g}$ ) and $r_{\infty}$ (instead of $x_{f}$ ) for the resulting background and foreground reflectances, respectively. The foreground reflectance $r_{\infty}$ namely corresponds to the reflectance for a layer with semi-infinite thickness. We also introduce $r_{1}$ and $t_{1}$, the reflectance and transmittance of a layer with unit thickness.

We use a two-flux method as the one proposed by Kubelka in 1954 [11]. It relies on compositions of layers, each one characterized its spectral reflectance and transmittance (assumed similar on both sides), and models the multiple reflections of diffuse light between them. This approach can be generalized to predict the reflectance $r_{N}$ and transmittance $t_{N}$ of a homogenous translucent layer of thickness $N$ by considering it as the superposition of $N$ identical sub-layers of unit thickness and defined by their reflectance $r_{1}$ and transmittance $t_{1}$ (Figure 6):

$$
\left\{\begin{array}{l}
r_{N}=\left(a-b\left(1-\frac{2}{1-\left(\frac{1-(a+b) r_{1}}{1-(a-b) r_{1}}\right)}\right)\right)^{-1} \\
t_{N}=\frac{2 b t_{1}^{N}}{(a+b)\left(1-(a-b) r_{1}\right)^{N}-(a-b)\left(1-(a+b) r_{1}\right)^{N}}
\end{array}\right.
$$

with

$a=\frac{1+r_{1}^{2}-t_{1}^{2}}{2 r_{1}}$ and $b=\sqrt{a^{2}-1}$

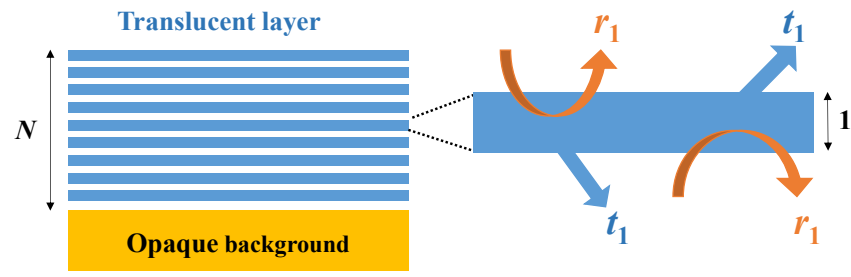

Figure 6. Homogenous translucent layer of thickness $N$ described as the superposition of $N$ identical sub-layers of unit thickness. Two-flux transfers for one unit thickness sub-layer defined by its reflectance $r_{1}$ and transmittance $t_{1}$. 
The complete demonstration is given by Hébert et al. [12]. It is worth noting that Eq. (15) can be applied for any positive real value of $N$ (and not only integer values). When many slices are stacked with each other, the stack reflectance reaches the invariant value $r_{\infty}$ for the opaque foreground layer (thickness $N \rightarrow \infty$ ):

$$
r_{\infty}=a-b
$$

When the translucent layer of thickness $N$ is on a background of reflectance $r_{g}$, the resulting reflectance $r$ can be obtained by applying the two-flux method again:

$$
r=r_{N}+\frac{r_{g} t_{N}^{2}}{1-r_{N} r_{g}}
$$

In contrast with the color mixing laws previously presented in this article, the two-flux method requires two spectral responses in order to completely characterize the scattering foreground layer. For example, $r_{N}$ and $t_{N}$ must be known to calculate the reflectance $r$ according to Eq. (17). But, in order to have a similar configuration as the previous approaches, the user has to choose the opaque foreground reflectance $r_{\infty}$. Since it is probably easier to imagine the reflectance than the transmittance of the layer, the second spectral response is the unit thickness reflectance $r_{1}$, assumed to be:

$$
r_{1}=\alpha r_{\infty}+\beta
$$

where $\alpha$ and $\beta$ are independent of the spectral band.

The parameter $\beta$ is the achromatic component of the unit thickness reflectance as often observed in case of surface scattering. As the reflectance $r_{1}$ must satisfy condition (1), it induces some restrictions for the values of $\alpha$ and $\beta$ :

$$
\left\{\begin{array}{l}
0 \leq \alpha<\frac{1-\beta}{r_{\infty}} \\
0 \leq \beta<1-\alpha r_{\infty}
\end{array}\right.
$$

The software automatically ensures that conditions (19) are satisfied. Finally, the user chooses first $r_{\infty}$ and then both constants $\alpha$ and $\beta$. The reflectance $r_{1}$ is calculated with the assumption (18) and the transmittance $t_{1}$ is deduced by the relation:

$$
a=\frac{1+r_{\infty}^{2}}{2 r_{\infty}} \text { and } t_{1}=\sqrt{1+r_{1}^{2}-2 r_{1} a}
$$

The resulting reflectance $r$ is finally calculated by applying equation (17) with relations (15). Figure 7 illustrates how the color haze due to scattering can be controlled with the parameters $\alpha$ and $\beta$.

\section{Inverse transparency}

We define the inverse transparency as the process that allows removing a transparency effect. In a formal way, it consists in calculating the background reflectance $x_{g}$ knowing the foreground reflectance $x_{f}$ for a given transparency rate $c$. Unfortunately, the additive-subtractive law (Eq. (6)) as well as the subtractive-additive law (Eq. (7)) cannot be easily inverted. However, for the generalized $f$-mean (Eq. (8)), the inversion is straightforward:
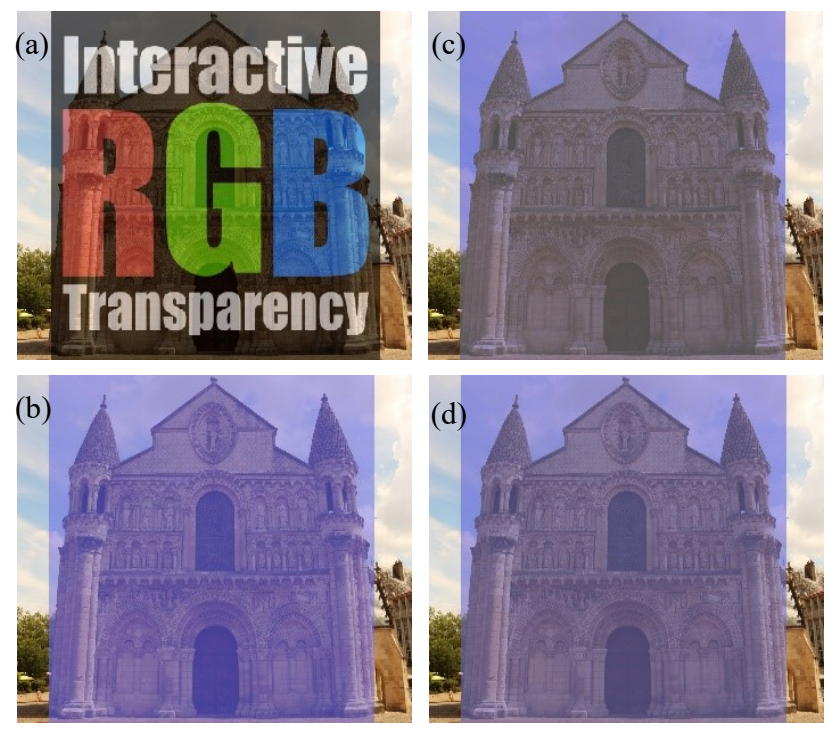

Figure 7. Translucent unit thickness layer on a background [Eqs. (15)-(20)] with $N=1$. (a) and (b) $\alpha=0.6, \beta=0$; (c) $\alpha=0, \beta=0.3$; (d) $\alpha=0.3, \beta=0.15$.

$x_{g}=f^{-1}\left(\frac{f(x)-(1-c) f\left(x_{f}\right)}{c}\right)$

The inverse transparency is of course possible if the foreground layer is not opaque $(c \neq 0)$.

When considering a translucent scattering layer on an opaque background, the equation (17) must be inverted:

$$
r_{g}=\frac{r-r_{N}}{t_{N}^{2}+r_{N}\left(r-r_{N}\right)}
$$

In the software, we propose two solutions. In the case of "inverse uniform transparency", the operation is performed with the same transparency rate for all selected pixels. The pixels for which the calculated background reflectance does not satisfy condition (1) are invalid and can be highlighted in a color selected by the user in the settings. For the "inverse non-uniform transparency", the operation is performed separately for each pixel. A parameter allows monitoring the removal of the foreground layer. The maximum removal corresponds to the limit before the pixel becomes invalid. An option allows visualizing the non-uniform removal part on a chosen background. Alternatively, the user can select zones and then the expected background color for these zones. The software computes the foreground color which, when applied to the average pixel color of the selected zones, ensures the best match with the color selected by the user.

Figure 8 illustrates the virtual varnish removal obtained by inverse uniform transparency on a JPEG image of Mona Lisa. The subtractive color mixing law is used with a yellow foreground layer for the varnish. Even the resulting image seems to be realistic, one should remember that this is the result of an empirical operation. The prediction of a painting visual appearance after varnish removal is much more complex and strongly depends on the work of art [13]. 

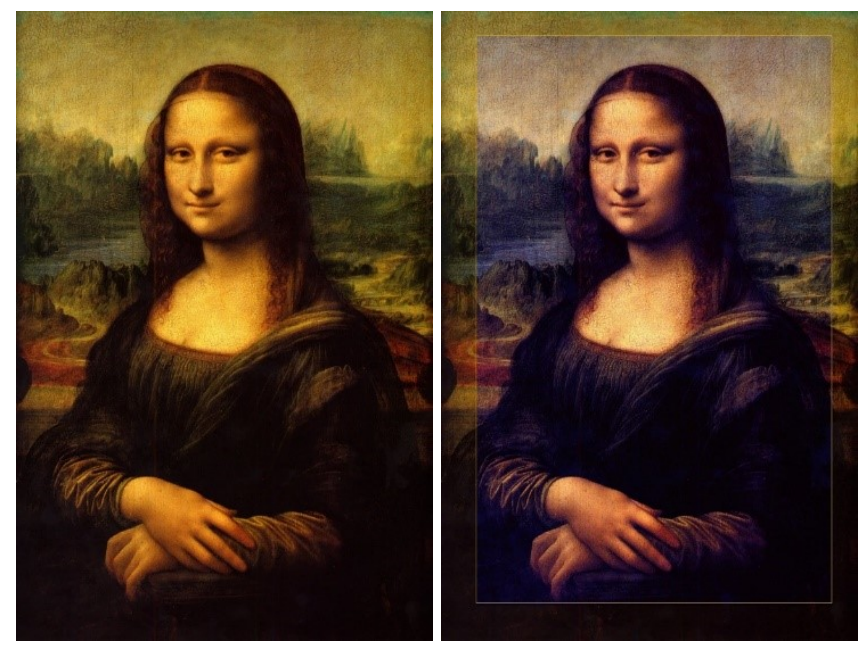

Figure 8. Illustration of a virtual varnish removal from a JPEG image (http://www.monalisa-davinci.com/images/Mona_Lisa.jpg), obtained by inversing the subtractive transparency law.

\section{Conclusion}

The software deals with RGB images but it could be directly generalized to multi- or hyper-spectral imaging. The transparency illusion is performed as a binary color mixing, but it can be also extended to mixing between any number of primaries or any number of translucent layers. One limitation is that the software does not simulate possible spatial modifications (blurring) of the image of the background due to a translucent layer. A spatial low-pass filter should be supplementary applied to mimic blurring.

Interactive $R G B$ Transparency can be freely downloaded [5]. Moreover, it is an open source software. Therefore, it offers the users to test and propose new color mixing laws to create transparency illusions.

\section{Acknowledgements}

The authors want to thank Andy Poudret and Florian Nadaud, students in informatics in the University of Poitiers. They developed the software in Java.

\section{References}

[1] J. Albers, Interaction of color, New Haven: Yale University Press, 1975.

[2] F. Metelli, "The perception of transparency," Scientific American 230, 91-98 (1974).

[3] M. D'Zmura, P. Colantoni, K. Knoblauch, B. Laget, "Color transparency," Perception 26, 471-492 (1997).

[4] L. Simonot, M. Hébert, "Between additive and subtractive color mixings: intermediate mixing models," JOSA A 31, 58-66 (2014).

[5] http://rgbtransparency.edel.univ-poitiers.fr/

[6] J. Yule, W. Nielsen, "The penetration of light into paper and its effect on halftone reproduction," in Proc. TAGA 3, 65-76 (1951).

[7] J. A. S Viggiano, "The Color of Halftone Tints," Proc. TAGA 37, 647-661 (1985)
[8] A. Lewandowski, M. Ludl, G. Byrne, G. Dorffner, "Applying the Yule-Nielsen equation with negative n," J. Opt. Soc. Am. A 23, 18271834 (2006).

[9] P. Kubelka, F. Munk, "Ein Beitrag zur Optik der Farbanstriche," Zeitschrift für technische Physik 12, 593-601 (1931).

[10] D. Duncan, "The colour of pigment mixtures," Journal of Oil Colour Chemistry Association 32, 296-321 (1949).

[11] P. Kubelka, "New contributions to the optics of intensively light scattering material, part II: Non-homogeneous layers," J. Opt. Soc. Am. 44, 330-335 (1954)

[12] M. Hébert, S. Mazauric, L. Simonot, Assessing the capacity of twoflux models to predict the spectral properties of layered materials, Proc. of IS\&T Electronic Imaging Symposium, Measuring, modeling, reproduction materials appearance, San Francisco (USA) 14-18 February 2016, 10pp.

[13] G. Trumpy, D. Conover, L. Simonot, M. Thoury, M. Picollo, J.K. Delaney, Experimental study on merits of virtual cleaning of paintings with aged varnish, Opt. Express 23 (2015) 33836-33848.

\section{Author Biography}

Lionel Simonot completed his PhD studies at the Centre de Recherche et de Restauration des Musées de France (C2RMF), located in the Palais du Louvre in Paris, where he studied the light scattering by painting glazes. He obtained the doctor grade in 2002. Since 2003, he is assistant professor at the Institut Pprime (CNRS UPR 3346 and University of Poitiers). His research activities focus on the optical properties of materials at different scales (nanocomposite layers, scattering layers, flat or rough interfaces).

Mathieu Hébert completed his PhD studies at the École Polytechnique Fédérale de Lausanne (EPFL, Switzerland) and obtained the doctor grade in 2006. Since 2010, he is assistant professor at the Institut d'OptiqueGraduate School and at the Laboratoire Hubert Curien of CNRS and University Jean Monnet of Saint-Etienne. His research activity is focused on optical models for predicting the visual rendering of colored surfaces. 